\title{
Hofer's symplectic energy and lagrangian intersections in contact geometry
}

\author{
By
}

Manabu AKAно

\begin{abstract}
There is a version of Lagrangian intersection theory in contact geometry [2]. But it works well only with very restrictive contact manifolds. For example, it does not work well with overtwisted contact 3-manifolds. Here we show the following. If we have an estimate on Hamiltonian functions of contact flow, then we can apply the theory to a much wider class of contact manifolds.
\end{abstract}

\section{Introduction}

In this paper, we show a version of Lagrangian intersection theory in contact geometry. Especially under an estimate of Hamiltonian functions of contact flow, we can construct Floer homology. Then we can show a version of the Arnold conjecture in a wide class of contact manifolds under the new condition.

Let $M$ be a $(2 n+1)$-dimensional contact manifold, i.e., $M$ has a 1 -form $\gamma$ which satisfies the condition $\gamma \wedge(d \gamma)^{n} \neq 0$. For simplicity, we consider the case of a global 1-form $\gamma$. Then the hyperplane distribution, $\xi$, defined by the kernel of $\gamma$ is called a contact structure on $M$, and the 1 -form $\gamma$ is called a contact form. For each contact form $\gamma$ there is a unique vector field $Y$ which satisfies the conditions $\iota(Y) \gamma=1$ and $\iota(Y) d \gamma=0$. We call this vector field $Y$ the Reeb vector field of $\gamma$.

We may regard the distribution $\xi$ as a rank 2 vector subbundle of $T M$, the tangent bundle of $M$. Then the restriction of $d \gamma$ on $\xi,\left.d \gamma\right|_{\xi}$, defines a non-degenerate 2 -form on $\xi$. Hence we can define a complex structure $J$ and a Hermitian metric on $\xi$ which has $\left.d \gamma\right|_{\xi}$ as a fundamental 2-form.

Next we can associate a symplectic manifold with a contact manifold $M$, so called the symplectization of $M$. Consider a product $R \times M$. Denote the pull-back of $\gamma$ by the projection from $R \times M$ to $M$, also by $\gamma$, and denote the coordinate of $R$ by $\theta$. Then a 2 -form $d\left(e^{\theta} \gamma\right)$ is an exact symplectic form on $R \times M$. And we define an almost complex structure $\tilde{J}$ on $R \times M$ by the following.

$$
\left.\tilde{J}\right|_{\xi}:=J, \quad \tilde{J} \frac{\partial}{\partial \theta}:=Y \quad \text { and } \quad \tilde{J} Y:=-\frac{\partial}{\partial \theta},
$$

Communicated by Prof. K. Fukaya, September 1, 2000 
where the pull-backs by the projection are also denoted by the same notation.

Now we introduce some important objects in this paper. Let $L_{1}$ be an $n$-dimensional submanifold of $M$ which satisfies the condition that the tangent bundle of $L_{1}$ is contained in the $\xi$, i.e., $T L_{1} \subset \xi$. Then we call such a submanifold a Legandrian submanifold. It is easy to see that $(n+1)$-dimensional submanifold $R \times L_{1}$ is a Lagrangian submanifold in the symplectization of $M$. Let $L_{0}$ be an $(n+1)$-dimensional submanifold of $M$. If there is a Lagrangian submanifold $\widehat{L_{0}}$ of the $R \times M$ which satisfies the condition that $\widehat{L_{0}}$ is diffeomorphic to $L_{0}$ by the projection, then we call $L_{0}$ a pre-Lagrangian submanifold and call $\widehat{L_{0}}$ a Lagrangian lift of $L_{0}$. A shift of the $\widehat{L_{0}}$ along the $R$-direction is also a Lagrangian lift of $L_{0}$. Then we define the following quantity and call it the height of $L_{0}$.

$$
\left.h_{\gamma}\left(\widehat{L_{0}}\right):=\right] \max _{p \in \widehat{L_{0}}} \theta(p)-\min _{p \in \widehat{L_{0}}} \theta(p),
$$

where $\theta$ is the coordinate of $R$ of the symplectization $R \times M$. Moreover we put $h_{\gamma}\left(L_{0}\right)$ to be the infimum of $h_{\gamma}\left(\widehat{L_{0}}\right)$ over the all Lagrangian lifts and call it the height of $L_{0}$.

Here we fix a contact form $\gamma$ of a contact structure $\xi$. For any function $H: M \rightarrow R$, there is a unique vector field $X_{H}$ which satisfies the conditions

$$
\iota\left(X_{H}\right) \gamma=-H \quad \text { and } \quad \iota\left(X_{H}\right) d \gamma=d H-(\iota(Y) d H) \gamma,
$$

where $Y$ is the Reeb vector field of $\gamma$. It is easy to see that the $X_{H}$ is a contact vector field, i.e., the flow generated by $X_{H}$ preserves the contact structure $\xi$. This map, from the space of functions to the space of contact vector fields, is bijective. Moreover it holds for time-dependent functions and time-dependent contact vector fields. Let $X_{H_{s}}$ be the contact vector field generated by a timedependent function $H_{s}$. We define a function $e^{\theta} H_{s}: R \times M \rightarrow R$ by $(\theta, q) \mapsto$ $e^{\theta} H_{s}(q)$. Then the Hamiltonian vector field generated by the $e^{\theta} H_{s}$ on the symplectization of $M$ is

$$
(\iota(Y) d H) \frac{\partial}{\partial \theta}+X_{H_{s}},
$$

where we regard $X_{H_{s}}$ as a vector field on the symplectization of $M$ which is constant along the $R$-direction.

Finally we introduce some quantities. Let $\gamma$ be a contact form, $Y$ the Reeb vector field of $\gamma$ and $L_{1}$ a Legandrian submanifold. We put

$$
\sigma_{\gamma}:=\inf \left\{\int_{l} \gamma \mid l \text { is a contractible closed orbit of } Y\right\} .
$$

If there is no contractible closed orbit, we put $\sigma_{\gamma}:=\infty$.

$$
\sigma_{\gamma}\left(L_{1}\right):=\inf \left\{\int_{l} \gamma \mid \begin{array}{c}
l \text { is an orbit of } Y \text { which satisfies that } l(0), l(1) \in L_{1} \\
\text { and } l \text { represents the zero element of } \pi_{1}\left(M, L_{1}\right)
\end{array}\right\} .
$$


If there is no such an orbit as above, we put $\sigma_{\gamma}\left(L_{1}\right):=\infty$. We put

$$
C_{\gamma}\left(L_{1}\right):=\min \left\{\sigma_{\gamma}, \sigma_{\gamma}\left(L_{1}\right)\right\} .
$$

For time-dependent function $H_{s}$ on $M$, we put

$$
\begin{aligned}
\left\|H_{s}\right\| & :=\int_{0}^{1}\left\{\max _{p \in M} H_{s}(p)-\min _{p \in M} H_{s}(p)\right\} d s, \\
d_{\gamma} & :=\max _{s \in[0,1], p \in M}\left|d H_{s}(Y)\right|,
\end{aligned}
$$

where $Y$ is the Reeb vector field of $\gamma$.

Theorem 1.1. $\quad$ Let $M$ be a closed contact manifold with a contact form $\gamma$. Fix this contact form $\gamma$. Let $L_{0}$ be a closed pre-Lagrangian submanifold and $L_{1}$ a closed Legandrian submanifold which satisfy the conditions $L_{0} \supset L_{1}$ and the boundary homomorphism $\pi_{2}\left(M, L_{0}\right) \rightarrow \pi_{1}\left(L_{0}\right)$ is trivial.

Let $\varphi_{1}$ be a time-1 map of the contact flow generated by a time-dependent function $H_{s}$ such that $\varphi_{1}\left(L_{1}\right)$ intersects $L_{0}$ transversaly. Assume that the following estimate holds.

$$
\left\|H_{s}\right\| \cdot \exp \left\{2 d_{\gamma}+h_{\gamma}\left(L_{0}\right)+\varepsilon\right\}<C_{\gamma}\left(L_{1}\right) .
$$

Then we obtain the following estimate.

$$
\sharp\left\{L_{0} \cap \varphi_{1}\left(L_{1}\right)\right\} \geq \operatorname{rank} H_{*}\left(L_{1} ; Z_{2}\right) .
$$

Notice that we do not need a $C^{1}$-small estimate of $H_{s}$ except for the Reeb direction. Eliashberg, Hofer and Salamon constructed a version of Lagrangian intersection theory in contact geometry [2]. Their theorems work well only with restrictive contact manifolds. For example, overtwisted contact 3-manifolds are not suitable, see [2] and [4]. Their theorem is the case of $C_{\gamma}\left(L_{1}\right)=\infty$ in our theorem.

Theorem 1.2 (Eliashberg, Hofer and Salamon). $\quad$ Let $M$ be a closed contact manifold with a contact form $\gamma$. Fix this contact form $\gamma$. Let $L_{0}$ be a closed pre-Lagrangian submanifold and $L_{1}$ a closed Legandrian submanifold which satisfy the conditions $L_{0} \supset L_{1}$ and the boundary homomorphism $\pi_{2}\left(M, L_{0}\right) \rightarrow \pi_{1}\left(L_{0}\right)$ is trivial.

Let $\varphi_{1}$ be a time-1 map of the contact flow generated by a time-dependent function $H_{s}$ such that $\varphi_{1}\left(L_{1}\right)$ intersects $L_{0}$ transversaly. Assume that there is no contractible closed orbit of the Reeb flow $Y$ and there is no orbit of $Y$ which satisfies that $l(0), l(1) \in L_{1}$ and $l$ represents the zero element of $\pi_{1}\left(M, L_{1}\right)$. Then we obtain the following estimate.

$$
\sharp\left\{L_{0} \cap \varphi_{1}\left(L_{1}\right)\right\} \geq \operatorname{rank} H_{*}\left(L_{1} ; Z_{2}\right) .
$$

The key points of the proof of our theorem is to show the compactness of the moduli space of pseudo-holomorphic disks with an estimate of the energy 
and to construct the homomorphism between Floer homologies of different contactomorphisms by using the technique of Chekanov [1].

\section{Path spaces and functionals}

In this section, we introduce path spaces and functionals. From now on, we denote the symplectization of a contact manifold $M$ by $P$. Let $\widetilde{H}_{s}:[0,1] \times$ $P \rightarrow R$ be a time-dependent function on $P$ and $\widetilde{X}_{\widetilde{H}_{s}}$ the Hamiltonian venter field generated by the Hamiltonian function $\widetilde{H}_{s}$. Namely the vector field $\widetilde{X}_{\widetilde{H}_{s}}$ satisfies the condition that

$$
d \widetilde{H}_{s}=\omega\left(\cdot, \widetilde{X}_{\widetilde{H}_{s}}\right),
$$

where $\omega$ is the symplectic form on $P$. Moreover, we put $G^{s}: P \rightarrow P$ to be the time-s Hamiltonian flow generated by $\widetilde{X}_{\widetilde{H}_{s}}$. Namely $G^{s}$ satisfies the condition that

$$
\left\{\begin{aligned}
\frac{d}{d s} G^{s} & =\widetilde{X}_{\widetilde{H}_{s}} \circ G^{s} \\
G^{0} & =\operatorname{id} .
\end{aligned}\right.
$$

For a closed Lagrangian submanifold $\widehat{L_{0}}$ of $P$ and a closed Legandrian submanifold $L_{1}$ of $M$ satisfying that $\widehat{L_{0}} \cap\left(R \times L_{1}\right)$ is not empty, we put

$$
\Omega_{s}^{\prime}:=\left\{l:[0,1] \rightarrow P \mid l(0) \in R \times L_{1}, l(1) \in G^{s}\left(\widehat{L_{0}}\right)\right\} .
$$

Moreover we denote a component which contains a path $l(t):=G^{t s}\left(x_{0}\right)$, for $x_{0} \in \widehat{L_{0}} \cap\left(R \times L_{1}\right)$, by $\Omega_{s}$. Put $\Omega:=\bigcup_{s \in[0,1]}\left(s, \Omega_{s}\right)$ and, for a fixed path $l_{0} \in \Omega$,

$$
\widetilde{\Omega}:=\left\{\begin{array}{l|l}
l_{\tau}(t):[0,1] \times[0,1] \rightarrow P & \begin{array}{l}
l_{\tau=0}(t)=l_{0}(t), \\
l_{\tau}(0) \in R \times L_{1}, \text { and } l_{\tau}(1) \in G^{s(\tau)}\left(\widehat{L_{0}}\right)
\end{array}
\end{array}\right\} .
$$

Next we introduce a functional $F$ on $\widetilde{\Omega}$. For $\widetilde{l}:=l_{\tau}(t) \in \widetilde{\Omega}$, we put

$$
F(\widetilde{l}):=\int_{0}^{1} d \tau \int_{0}^{1} d t \omega\left(\frac{\partial}{\partial t} l, \frac{\partial}{\partial \tau} l\right)-\int_{s(0)}^{s(1)} d s(\tau) \widetilde{H}_{s(\tau)}\left(l_{\tau}(1)\right) .
$$

Lemma 2.1. The value of $F$ depends only on the homotopy type of $l$ which fixes an end path. Namely, put $\widetilde{l}_{\sigma}:[0,1] \mapsto \widetilde{\Omega} ; \sigma \rightarrow l(\sigma ; \tau, t)$ which satisfies that $l(\sigma ; 1, t)=l\left(\sigma^{\prime} ; 1, t\right)$, for any $\sigma, \sigma^{\prime} \in[0,1]$, then $F\left(\widetilde{l}_{\sigma=0}\right)=F\left(\widetilde{l}_{\sigma=1}\right)$.

Lemma 2.2. Let $L_{0}$ be a closed pre-Lagrangian submanifold of $M$ with a Lagrangian lift $\widehat{L_{0}}$ and $L_{1}$ be a closed Legandrian submanifold of $M$. Suppose that $L_{1}$ is contained in $L_{0}$, i.e., $L_{1} \subset L_{0}$, and the boundary homomorphism $\pi_{2}\left(M, L_{0}\right) \rightarrow \pi_{1}\left(L_{0}\right)$ is trivial. Then, for $\widetilde{l} \in \widetilde{\Omega}$ which satisfies that $\widetilde{l}_{\tau=1}=\widetilde{l}_{\tau=0}$, we obtain $F(\widetilde{l})=0$. 
From the above lemmas, we can regard the functional $F$ on the $\widetilde{\Omega}$ as a functional on the $\Omega$, i.e., the value of $F$ is determined by an end point $l_{\tau=1}$ and the base point $l_{0}$. Note that the restriction of $F$ on $\Omega_{s}$ coincides with the usual Floer's functional and denote $\left.F\right|_{\Omega_{s}}$ by $F_{s}$.

\section{Floer homology}

In this section, let $\tilde{J}$ be the almost complex structure on $P$ as mentioned in introduction. To put it more precisely, we have to consider perturbations of almost complex structures. It is a little complicated. Hence we omit it here. See [2].

We define a metric on $\Omega_{s}$ by

$$
\left(\xi_{1}, \xi_{2}\right):=\int_{0}^{1} \omega\left(\xi_{1}(t), \tilde{J} \xi_{2}(t)\right) d t,
$$

where $\xi_{1}, \xi_{2} \in T_{l} \Omega_{s}$. For this metric, the gradient vector field $\nabla F_{s}$ of the $F_{s}$ is

$$
\nabla F_{s}(l)(t)=\tilde{J}(l(t)) \dot{l}(t) .
$$

The set of critical points of $F_{s}$ consists of the intersection points of $R \times L_{1}$ and $G^{s}\left(\widehat{L_{0}}\right)$. Suppose that $G^{s}\left(\widehat{L_{0}}\right)$ intersects $R \times L_{1}$ transversaly. For critical points $x_{+}, x_{-}$of $F_{s}$, we put the moduli space of descending gradient trajectories as

$$
\begin{aligned}
& \mathcal{M}_{s}\left(x_{-}, x_{+}\right) \\
& :=\left\{\begin{array}{l|l}
u: R \rightarrow \Omega_{s} & \begin{array}{l}
\frac{d u(\tau)}{d \tau}=-\nabla F_{s}(u(\tau)), u \text { is not constant and } \\
\lim _{\tau \rightarrow \pm \infty} u(\tau)=x_{ \pm}
\end{array}
\end{array}\right\} .
\end{aligned}
$$

For a suitable perturbation of almost complex structures we can assume that the regularity condition holds and this space is a manifold, see [2]. And $R$ acts on $\mathcal{M}_{s}\left(x_{-}, x_{+}\right)$by translation, $u(\cdot) \mapsto u(\cdot+a), a \in R$. We denote the quotient by $\widehat{\mathcal{M}}_{s}\left(x_{-}, x_{+}\right)$.

From now on, let $\widehat{L_{0}}$ be the Lagrangian lift of a closed pre-Lagrangian submanifold $L_{0}$ with the condition min $\widehat{p}_{p \in \bar{L}_{0}} \theta(p)=0$ and $L_{1}$ be a closed Legandrian submanifold with the condition $L_{1} \subset L_{0}$. Moreover assume that the boundary homomorphism $\pi_{2}\left(M, L_{0}\right) \rightarrow \pi_{1}\left(L_{0}\right)$ is trivial. From these assumptions we can say the followings. First we may regard the functional $F$ on $\widetilde{\Omega}$ as a functional on $\Omega$ from Lemma 2.2. Second the bubbling off phenomena can't occur at the boundary points of pseudo-holomorphic disks. Because the symplectic form of $P$ is exact the bubbling off phenomena always can't occur at the interior points of pseudo-holomorphic disks.

Fix constants $b_{-}$and $C^{\prime}$. Let $p: R \rightarrow\left[b_{-}, b_{-}+C^{\prime}\right)$ be a projection. For a critical point $x$ of $F_{s}$, we put $\widetilde{F}_{s}(x):=p \circ F_{s}(x)$. Next we put the length of a descending gradient trajectory $u$ by

$$
l(u):=-\int_{-\infty}^{\infty} u^{*} d F_{s}=\int_{-\infty}^{\infty}\left(\frac{d u(\tau)}{d \tau}, \frac{d u(\tau)}{d \tau}\right) d \tau>0 .
$$


Then we define the moduli space of distinguished gradient trajectories by

$$
\mathcal{M}_{s}^{d}\left(x_{-}, x_{+}\right):=\left\{u \in \mathcal{M}_{s}\left(x_{-}, x_{+}\right) \mid l(u)=\widetilde{F}_{s}\left(x_{-}\right)-\widetilde{F}_{s}\left(x_{+}\right)\right\} .
$$

If $\widetilde{F}_{s}\left(x_{-}\right)-\widetilde{F}_{s}\left(x_{+}\right)$is negative, then $\mathcal{M}_{s}^{d}\left(x_{-}, x_{+}\right)$is empty. And the quotient of $\mathcal{M}_{s}^{d}\left(x_{-}, x_{+}\right)$by the action of $R$ is denoted by $\widehat{\mathcal{M}}_{s}^{d}\left(x_{-}, x_{+}\right)$.

Theorem 3.1. Assume that $C^{\prime} e^{d+\varepsilon}<C_{\gamma}\left(L_{1}\right)$ for some positive number $\varepsilon$, where $d=\int_{0}^{s} \max _{p \in G^{t}\left(\widehat{L}_{0}\right)}\left|d \theta\left(X_{\widetilde{H}_{t}}\right)\right| d t$. Then the images of all distinguished gradient trajectories are contained in some compact set of $P$.

Corollary 3.2. $\quad$ There is no bubble in the distinguished gradient trajectories.

Corollary 3.3. The set of isolated points of $\widehat{\mathcal{M}}_{s}^{d}\left(x_{-}, x_{+}\right)$is compact.

We show the proof of Theorem 3.1 in the last section. Owing to the compactness, we can define Floer homology. Let $Y(s)$ be the set of critical points of $F_{s}$ and $C(s)$ be the vector space over $Z_{2}$ spanned by elements of $Y(s)$. Then we define a boundary operator $\partial_{s}: C(s) \rightarrow C(s)$ by

$$
\partial_{s} x:=\sum_{y \in Y(s)} \sharp\left\{\text { isolated points of } \widehat{\mathcal{M}}_{s}^{d}(x, y)\right\} y,
$$

where $x \in Y(s)$.

Proposition 3.4. We have $\partial_{s}^{2}=0$.

Proof. We prove this proposition by the standard gluing argument in the Floer theory. From the definition

$$
\partial_{s}^{2} x:=\sum_{z, y \in Y(s)} \sharp\left\{\text { isolated points of } \widehat{\mathcal{M}}_{s}^{d}(x, y)\right\} \sharp\left\{\text { isolated points of } \widehat{\mathcal{M}}_{s}^{d}(y, z)\right\} z \text {. }
$$

Hence we show that the coefficient of each $z$ is even. Take isolated points $u_{1} \in \widehat{\mathcal{M}}_{s}^{d}(x, y)$ and $u_{2} \in \widehat{\mathcal{M}}_{s}^{d}(y, z)$, then there is a 1-dimensional component $N$ of $\widehat{\mathcal{M}}_{s}(x, z)$ so that $\left(u_{1}, u_{2}\right)$ is an end of the compactification of $N$. Because the length is additive under the gluing procedure, it holds that $l(u)=l\left(u_{1}\right)+l\left(u_{2}\right)$ $=\widetilde{F}_{s}(x)-\widetilde{F}_{s}(z)$ for $u \in N$. Then $N \subset \widehat{\mathcal{M}}_{s}^{d}(x, z)$. From Corollary 3.2 there is no bubble in the sequence of points of $N$. And there are isolated points $u_{1}^{\prime} \in \widehat{\mathcal{M}}_{s}\left(x, y^{\prime}\right)$ and $u_{2}^{\prime} \in \widehat{\mathcal{M}}_{s}\left(y^{\prime}, z\right)$ so that $\left(u_{1}^{\prime}, u_{2}^{\prime}\right)$ is the other end point of the compactification of $N$. Finally we show that $u_{1}^{\prime}$ and $u_{2}^{\prime}$ are distinguished. We put $l\left(u_{1}^{\prime}\right)=\widetilde{F}_{s}(x)-\widetilde{F}_{s}\left(y^{\prime}\right)+n C^{\prime}$ and $l\left(u_{2}^{\prime}\right)=\widetilde{F}_{s}\left(y^{\prime}\right)-\widetilde{F}_{s}(z)-n C^{\prime}, n \in Z$. Since $l\left(u_{1}^{\prime}\right)>0$ and $C^{\prime}>\widetilde{F}_{s}(x)-\widetilde{F}_{s}\left(y^{\prime}\right)>-C^{\prime}, n$ have to be non-negative. Similarly, since $l\left(u_{2}^{\prime}\right)>0$ and $C^{\prime}>\widetilde{F}_{s}\left(y^{\prime}\right)-\widetilde{F}_{s}(z)>-C^{\prime}, n$ have to be nonpositive. Then $n$ is zero and $l\left(u_{1}^{\prime}\right)=\widetilde{F}_{s}(x)-\widetilde{F}_{s}\left(y^{\prime}\right)$ and $l\left(u_{2}^{\prime}\right)=\widetilde{F}_{s}\left(y^{\prime}\right)-\widetilde{F}_{s}(z)$. 
Namely, $u_{1}^{\prime}$ and $u_{2}^{\prime}$ are distinguished. Hence the coefficient of each $z$ is even and $\partial_{s}^{2}=0$.

We construct a homology group $H\left(C(s), \partial_{s}\right)$ from this chain complex $\left(C(s), \partial_{s}\right)$. In the next section, we show the following. If we have a suitable estimate of a Hamiltonian function, then there is an injective homomorphism $V_{1}^{s}: H\left(C(s), \partial_{s}\right) \rightarrow H\left(C(1), \partial_{1}\right)$ for small $s$. Hence we have rank $H\left(C(1), \partial_{1}\right) \geq$ rank $H\left(C(s), \partial_{s}\right)$. Of course, if $R \times L_{1}$ intersects $G^{1}\left(\widehat{L_{0}}\right)$ transversaly, then $\sharp\left\{\left(R \times L_{1}\right) \cap G^{1}\left(\widehat{L_{0}}\right)\right\} \geq \operatorname{rank} H\left(C(1), \partial_{1}\right)$.

Proposition 3.5. For some small s, $H\left(C(s), \partial_{s}\right)$ is isomorphic to $H_{*}\left(L_{1}\right.$; $Z_{2}$ ) as a vector space.

Sketch of proof. There is a contact diffeomorphism from a small neighborhood of $L_{1}$ in $M$ to a small neighborhood of the zero section of 1-jet of $L_{1}$ such that the image of $L_{0}$ is the 0 -wall. Under the assumption that $\varphi_{s}\left(L_{1}\right)$ intersects $W$ transversaly, for small $s$ there is a Morse function such that the set of its critical points is isomorphic to the set of intersection points of $\varphi_{s}\left(L_{1}\right)$ and $W$. And take a suitable metric on $L_{1}$, the set of gradient trajectories of the Morse function between critical points $x_{-}$and $x_{+}$is isomorphic to the set of gradient trajectories of the Floer's functional between the intersection points $x_{-}$and $x_{+}$. Moreover for some small $s$ the gradient trajectories of the Floer's functional are distinguished. Then the Morse complex is isomorphic to the complex $\left(C(s), \partial_{s}\right)$.

Hence if we have a suitable estimate of Hamiltonian function, then we obtain $\sharp\left\{\left(R \times L_{1}\right) \cap G^{1}\left(\widehat{L_{0}}\right)\right\} \geq \operatorname{rank} H_{*}\left(L_{1} ; Z_{2}\right)$.

\section{Continuations and homotopy of continuations}

In this section, we describe the technique of Chekanov [1]. We put

$$
a_{+}:=\int_{0}^{1} \max _{p \in G^{s}\left(\widehat{L_{0}}\right)} \widetilde{H}_{s}(p) d s \text { and } a_{-}:=\int_{0}^{1} \min _{p \in G^{s}\left(\widehat{L_{0}}\right)} \widetilde{H}_{s}(p) d s,
$$

and we may think that $a_{-} \leq 0 \leq a_{+}$. From now we assume that $a_{+}-a_{-}<C^{\prime}$. At the beginning we can take a generic base point of $\widetilde{\Omega}$ and $\varepsilon>0$ such that $F_{s}(y)>0$ for any $s<\varepsilon$ and any $y \in Y(s)$. Moreover we retake the generic base point so that $\widetilde{F}_{1}(y) \neq a_{-}$for any $y \in Y(1)$. Since the number of elements of $Y(1)$ is finite we can choose the number $b_{-}$so that $\widetilde{F}_{1}(y) \notin\left[b_{-}, a_{-}\right]$for any $y \in Y(1)$. Then we take an interval $\left(c_{-}, c_{+}\right) \subset\left(b_{-}, b_{+}\right)$, where $b_{+}=b_{-}+C^{\prime}$, such that $a_{-}<c_{-}, a_{+}<c_{+}$and $\widetilde{F}_{1}(y) \in\left[c_{-}, c_{+}\right]$for any $y \in Y(1)$. Moreover we take $c_{-}$enough close to $a_{-}$and $c_{+}$enough close to $b_{+}$and retake $\varepsilon>0$ small such that $\widetilde{F}_{s}(x) \in\left[c_{-}-a_{-}, c_{+}-a_{+}\right]$for any $s<\varepsilon$ and $x \in Y(s)$.

We introduce a continuation map $Q_{s_{+}}^{s_{-}} \in \operatorname{Hom}\left(C\left(s_{-}\right), C\left(s_{+}\right)\right)$. Let $\rho: R \rightarrow$ $[0,1]$ be a function which satisfies that there are some constants $K>0$ such that 
$\rho(\tau)=s_{-}$for $\tau<-K$ and $\rho(\tau)=s_{+}$for $\tau>K$. We call such a function an $\left(s_{-}, s_{+}\right)$-continuation function. Moreover, if $\rho$ is a monotone function, we call it a monotone $\left(s_{-}, s_{+}\right)$-continuation function. For critical points $x_{-} \in Y\left(s_{-}\right)$ and $x_{+} \in Y\left(s_{+}\right)$, we put the moduli spaces of continuation trajectories as

$\mathcal{M}_{\rho}\left(x_{-}, x_{+}\right):=\left\{u: R \rightarrow \Omega \mid \frac{d u(\tau)}{d \tau}=-\nabla F_{\rho(\tau)}(u(\tau))\right.$ and $\left.\lim _{\tau \rightarrow \pm \infty} u(\tau)=x_{ \pm}\right\}$.

For a suitable perturbation of almost complex structures we can assume that the regularity condition holds and this space is manifold.

For a continuation trajectory $u$, we put the length $l(u)$ by

$$
l(u):=-\int_{-\infty}^{\infty} u^{*} d F
$$

and the symplectic area $A(u)$ by

$$
A(u):=-\int_{R \times[0,1]} u^{*} \omega=\int_{-\infty}^{\infty}\left(\frac{d u(\tau)}{d \tau}, \frac{d u(\tau)}{d \tau}\right) d \tau \geq 0 .
$$

And we put

$$
h(u):=\int_{-\infty}^{\infty} \frac{d \rho(\tau)}{d \tau} \widetilde{H}_{\rho(\tau)}(u(\tau, 1)) d \tau,
$$

then $A(u)=l(u)+h(u)$. We define the moduli space of distinguished continuation trajectories by

$$
\mathcal{M}_{\rho}^{d}\left(x_{-}, x_{+}\right):=\left\{u \in \mathcal{M}_{\rho}\left(x_{-}, x_{+}\right) \mid l(u)=\widetilde{F}_{s_{-}}\left(x_{-}\right)-\widetilde{F}_{s_{+}}\left(x_{+}\right)\right\} .
$$

Lemma 4.1. Let $s_{-}<\varepsilon, s_{+}=1$ or $s_{-}=1, s_{+}<\varepsilon$. And let $\rho$ be a monotone $\left(s_{-}, s_{+}\right)$-continuation function, then we obtain

$$
A(u) \leq c_{+}-c_{-}\left(<C^{\prime}\right),
$$

where $u \in \mathcal{M}_{\rho}^{d}\left(x_{-}, x_{+}\right)$.

Proof. First let $s_{-}<\varepsilon$ and $s_{+}=1$. Notice that $\rho$ is monotone. We obtain

$$
\begin{aligned}
h(u) & \leq \int_{-\infty}^{\infty} \frac{d \rho(\tau)}{d \tau} \max _{p \in G^{\rho(\tau)}\left(\widehat{L_{0}}\right)} \widetilde{H}_{\rho(\tau)}(p) d \tau \\
& =\int_{s}^{1} \max _{p \in G^{s}\left(\widehat{L_{0}}\right)} \widetilde{H}_{s}(p) d s \leq a_{+},
\end{aligned}
$$

where $u \in \mathcal{M}_{\rho}^{d}\left(x_{-}, x_{+}\right)$. Since $l(u)=\widetilde{F}_{s_{-}}\left(x_{-}\right)-\widetilde{F}_{s_{+}}\left(x_{+}\right) \leq\left(c_{+}-a_{+}\right)-c_{-}$, we obtain $A(u)=l(u)+h(u) \leq c_{+}-c_{-}$. 
Second let $s_{-}=1$ and $s_{+}<\varepsilon$. Notice that $\rho$ is monotone. We obtain

$$
\begin{aligned}
h(u) & \leq \int_{-\infty}^{\infty} \frac{d \rho(\tau)}{d \tau} \min _{p \in G^{\rho(\tau)}\left(\widehat{L_{0}}\right)} \widetilde{H}_{\rho(\tau)}(p) d \tau \\
& =\int_{1}^{s} \min _{p \in G^{s}\left(\widehat{L_{0}}\right)} \widetilde{H}_{s}(p) d s \leq-a_{-},
\end{aligned}
$$

where $u \in \mathcal{M}_{\rho}^{d}\left(x_{-}, x_{+}\right)$. Since $l(u)=\widetilde{F}_{s_{-}}\left(x_{-}\right)-\widetilde{F}_{s_{+}}\left(x_{+}\right) \leq c_{+}-\left(c_{-}-a_{-}\right)$, we obtain $A(u)=l(u)+h(u) \leq c_{+}-c_{-}$.

Theorem 4.2. $\quad$ Let $s_{-}, s_{+}$and $\rho$ be the same as Lemma 4.1. Assume that $C^{\prime} e^{d+\varepsilon}<C_{\gamma}\left(L_{1}\right)$ for some positive number $\varepsilon$, where $d=\int_{0}^{1} \max _{p \in G^{t}\left(\widehat{L_{0}}\right)}$ $\left|d \theta\left(X_{\widetilde{H}_{t}}\right)\right| d t$. Then the images of all continuation trajectories are contained in some compact set of $P$.

Corollary 4.3. $\quad$ There is no bubble in the continuation trajectories.

The proofs of them is the same ones as Theorem 3.1 and Corollary 3.2.

For a monotone $\left(s_{-}, s_{+}\right)$-continuation function $\rho$, we define a continuation $\operatorname{map} Q_{s_{-}}^{s_{+}}: C\left(s_{-}\right) \rightarrow C\left(s_{+}\right)$by

$$
Q_{s_{-}}^{s_{+}}(x):=\sum_{y \in Y\left(s_{+}\right)} \sharp\left\{\text { isolated points of } \mathcal{M}_{\rho}^{d}(x, y)\right\} y,
$$

where $x \in Y\left(s_{-}\right)$.

Proposition 4.4. Let $s_{-}<\varepsilon, s_{+}=1$ or $s_{-}=1, s_{+}<\varepsilon$. And let $\rho$ be a monotone $\left(s_{-}, s_{+}\right)$-continuation function, then we obtain

$$
Q_{s_{+}}^{s_{-}} \circ \partial_{s_{-}}=\partial_{s_{+}} \circ Q_{s_{+}}^{s_{-}} .
$$

Proof. Let $x \in Y\left(s_{-}\right)$and $z \in Y\left(s_{+}\right)$. Take a pair of isolated points $\left(u_{1}, u_{2}\right)$ where $u_{1} \in \widehat{\mathcal{M}}_{s_{-}}^{d}(x, y), u_{2} \in \mathcal{M}_{\rho}^{d}(y, z), y \in Y\left(s_{-}\right)$or $u_{1} \in \mathcal{M}_{\rho}^{d}(x, y)$, $u_{2} \in \widehat{\mathcal{M}}_{s_{+}}^{d}(y, z), y \in Y\left(s_{+}\right)$. Then there is a 1-dimensional component $N$ of $\mathcal{M}_{\rho}(x, z)$ so that $\left(u_{1}, u_{2}\right)$ is an end of the compactification of $N$. Because the length is additive under the gluing procedure, it holds that $l(u)=l\left(u_{1}\right)+$ $l\left(u_{2}\right)=\widetilde{F}_{s_{-}}(x)-\widetilde{F}_{s_{+}}(z)$ for $u \in N$. Then $N \subset \mathcal{M}_{\rho}^{d}(x, z)$. From Corollary 4.3 there is no bubble in the sequence of points of $N$. And there is a pair of isolated points $\left(u_{1}^{\prime}, u_{2}^{\prime}\right)$ where $u_{1}^{\prime} \in \widehat{\mathcal{M}}_{s_{-}}(x, y), u_{2}^{\prime} \in \mathcal{M}_{\rho}(y, z), y \in Y\left(s_{-}\right)$or $u_{1}^{\prime} \in \mathcal{M}_{\rho}(x, y), u_{2}^{\prime} \in \widehat{\mathcal{M}}_{s_{+}}(y, z), y \in Y\left(s_{+}\right)$so that $\left(u_{1}^{\prime}, u_{2}^{\prime}\right)$ is the other end point of the compactification of $N$. Finally we need to show that $u_{1}^{\prime}$ and $u_{2}^{\prime}$ are distinguished.

Lemma 4.5. If $u \in \mathcal{M}_{\rho}\left(x_{-}, x_{+}\right)$, then we have $l(u) \geq \widetilde{F}_{s_{-}}\left(x_{-}\right)-$ $\widetilde{F}_{s_{+}}\left(x_{+}\right)$. 
Proof. First let $s_{-}<\varepsilon$ and $s_{+}=1$. Then we have

$$
\begin{aligned}
l(u)=A(u)-h(u) & \geq-a_{+} \\
& >\left(c_{+}-a_{+}\right)-c_{-}-C^{\prime} \\
& \geq \widetilde{F}_{s_{-}}\left(x_{-}\right)-\widetilde{F}_{s_{+}}\left(x_{+}\right)-C^{\prime} .
\end{aligned}
$$

Second let $s_{-}=1$ and $s_{+}<\varepsilon$. Then we have

$$
\begin{aligned}
l(u)=A(u)-h(u) & \geq a_{-} \\
& >c_{+}-\left(c_{-}-a_{-}\right)-C^{\prime} \\
& \geq \widetilde{F}_{s_{-}}\left(x_{-}\right)-\widetilde{F}_{s_{+}}\left(x_{+}\right)-C^{\prime} .
\end{aligned}
$$

In both cases, we obtain $l(u)>\widetilde{F}_{s_{-}}\left(x_{-}\right)-\widetilde{F}_{s_{+}}\left(x_{+}\right)-C^{\prime}$. Moreover, $l(u)=$ $\widetilde{F}_{s_{-}}\left(x_{-}\right)-\widetilde{F}_{s_{+}}\left(x_{+}\right)+n C^{\prime}, n \in Z$. Hence we obtain $l(u)=\widetilde{F}_{s_{-}}\left(x_{-}\right)-\widetilde{F}_{s_{+}}\left(x_{+}\right)$.

Let $\left(u_{1}, u_{2}\right) \in \widehat{\mathcal{M}}_{s_{-}}(x, y) \times \mathcal{M}_{\rho}(y, z), y \in Y\left(s_{-}\right)$, be an end point of the compactification of a 1-dimensional component $N \subset \mathcal{M}_{\rho}^{d}(x, z)$. Put $l\left(u_{1}\right)=$ $\widetilde{F}_{s_{-}}(x)-\widetilde{F}_{s_{-}}(y)+n C^{\prime}$ and $l\left(u_{2}\right)=\widetilde{F}_{s_{-}}(y)-\widetilde{F}_{s_{+}}(z)-n C^{\prime}$. Because $l\left(u_{1}\right)=$ $A\left(u_{1}\right) \geq 0$, we obtain $n \geq 0$. From Lemma $4.5, l\left(u_{2}\right) \geq \widetilde{F}_{s_{-}}(y)-\widetilde{F}_{s_{+}}(z)$, we obtain $n \leq 0$. Hence $n=0$ and $u_{1}, u_{2}$ are distinguished. Similarly, let $\left(u_{1}, u_{2}\right) \in$ $\mathcal{M}_{\rho}(x, y) \times \widehat{\mathcal{M}}_{s_{+}}(y, z), y \in Y\left(s_{+}\right)$, be an end point of the compactification of a 1-dimensional component $N \subset \mathcal{M}_{\rho}^{d}(x, z)$. Put $l\left(u_{1}\right)=\widetilde{F}_{s_{-}}(x)-\widetilde{F}_{s_{+}}(y)+n C^{\prime}$ and $l\left(u_{2}\right)=\widetilde{F}_{s_{+}}(y)-\widetilde{F}_{s_{+}}(z)-n C^{\prime}$. Because $l\left(u_{2}\right)=A\left(u_{2}\right) \geq 0$, we obtain $n \leq 0$. From Lemma $4.5, l\left(u_{1}\right) \geq \widetilde{F}_{s_{+}}(x)-\widetilde{F}_{s_{+}}(y)$, we obtain $n \geq 0$. Hence $n=0$ and $u_{1}, u_{2}$ are distinguished. Then we obtain

$$
\left(Q_{s_{+}}^{s_{-}} \circ \partial_{s_{-}}-\partial_{s_{+}} \circ Q_{s_{+}}^{s_{-}}\right) x=0
$$

where $x \in Y\left(s_{-}\right)$.

From $Q_{1}^{s}$ and $Q_{s}^{1}, s<\varepsilon$, we construct homomorphisms

$$
V_{1}^{s}: H\left(C(s), \partial_{s}\right) \rightarrow H\left(C(1), \partial_{1}\right) \text { and } V_{s}^{1}: H\left(C(1), \partial_{1}\right) \rightarrow H\left(C(s), \partial_{s}\right) .
$$

Proposition 4.6. We have $V_{s}^{1} \circ V_{1}^{s}=\mathrm{id}$.

Proof. Consider a family of $(s, s)$-continuation functions $\pi_{\omega}, \omega \in[0, \infty)$, which satisfies the following conditions.

- $\pi_{0}(\tau) \equiv s$.

- $\omega \mapsto \pi_{\omega}(0)$ is monotone and surjective onto $[s, 1]$.

- $d \pi_{\omega}(\tau) / d \tau \geq 0$, for $\tau<0$, and $d \pi_{\omega}(\tau) / d \tau \leq 0$, for $\tau>0$.

- For large $\omega$,

$$
\pi_{\omega}(\tau)= \begin{cases}\rho^{-}(\tau+\omega) & \text { for } \tau \leq 0 \\ \rho^{+}(\tau-\omega) & \text { for } \tau \geq 0\end{cases}
$$


where $\rho^{+}$and $\rho^{-}$are monotone $(1, s)$-continuation function and monotone $(s, 1)$-continuation function which we use to construct $Q_{s}^{1}$ and $Q_{1}^{s}$ respectively. For critical points $x_{-}$and $x_{+} \in Y(s)$, we put

$$
\mathcal{M}_{\pi}\left(x_{-}, x_{+}\right):=\left\{(\omega, u) \mid u \in \mathcal{M}_{\pi_{\omega}}\left(x_{-}, x_{+}\right)\right\} .
$$

For a suitable perturbation of almost complex structures we can assume that the regularity condition holds and this space is manifold. Moreover we put

$$
\mathcal{M}_{\pi}^{d}\left(x_{-}, x_{+}\right):=\left\{(\omega, u) \mid u \in \mathcal{M}_{\pi_{\omega}}^{d}\left(x_{-}, x_{+}\right)\right\} .
$$

Lemma 4.7. Let $u \in \mathcal{M}_{\pi}^{d}\left(x_{-}, x_{+}\right)$, then we obtain

$$
A(u) \leq c_{+}-c_{-}\left(<C^{\prime}\right) .
$$

Proof. For $u \in \mathcal{M}_{\pi_{\omega}}^{d}\left(x_{-}, x_{+}\right)$,

$$
\begin{aligned}
h(u) \leq & \int_{-\infty}^{0} \frac{d \pi_{\omega}(\tau)}{d \tau} \max _{p \in G^{\pi_{\omega}(\tau)}\left(\widehat{L_{0}}\right)} \widetilde{H}_{\pi_{\omega}(\tau)}(p) d \tau \\
& +\int_{0}^{\infty} \frac{d \pi_{\omega}(\tau)}{d \tau} \min _{p \in G^{\pi_{\omega}(\tau)}\left(\widehat{L_{0}}\right)} \widetilde{H}_{\pi_{\omega}(\tau)}(p) d \tau \\
= & \int_{s}^{0} \max _{p \in G^{s}\left(\widehat{L_{0}}\right)} \widetilde{H}_{s}(p) d s+\int_{0}^{s} \min _{p \in G^{s}\left(\widehat{L}_{0}\right)} \widetilde{H}_{s}(p) d s \\
\leq & a_{+}-a_{-} .
\end{aligned}
$$

Since $l(u)=\widetilde{F}_{s}\left(x_{-}\right)-\widetilde{F}_{s}\left(x_{+}\right) \leq\left(c_{+}-a_{+}\right)-\left(c_{-}-a_{-}\right)$, we obtain $A(u)=$ $l(u)+h(u) \leq c_{+}-c_{-}$.

From Lemma 4.7 the set of isolated points of $\mathcal{M}_{\pi}^{d}(x, z)$ is compact and there is no bubble in $\mathcal{M}_{\pi}^{d}(x, z)$ in the same way of Corollary 4.3. Hence the number of 1-dimensional components of $\mathcal{M}_{\pi}^{d}(x, z)$ is finite. And there are four types of the end points of the compactification of a 1-dimensional component as follows.

1. a pair $\left(u_{1}, u_{2}\right)$ of isolated points $u_{1} \in \mathcal{M}_{\rho^{-}}(x, y)$ and $u_{2} \in \mathcal{M}_{\rho^{+}}(y, z)$, for $y \in Y(1)$.

2. an isolated point $u \in \mathcal{M}_{\pi_{0}}(x, z)$.

3. a pair $\left(u_{1}, u_{2}\right)$ of isolated points $u_{1} \in \widehat{\mathcal{M}}_{s}(x, y)$ and $u_{2} \in \mathcal{M}_{\pi_{\omega}}(y, z)$, for $y \in Y(s)$.

4. a pair $\left(u_{1}, u_{2}\right)$ of isolated points $u_{1} \in \mathcal{M}_{\pi_{\omega}}(x, y)$ and $u_{2} \in \widehat{\mathcal{M}}_{s}(y, z)$, for $y \in Y(s)$.

We put

$$
h_{s}(x):=\sum_{y \in Y(s)} \sharp\left\{\text { isolated points of } \mathcal{M}_{\pi}^{d}(x, y)\right\} y,
$$

where $x \in Y(s)$. If all the end points as mentioned are distinguished, we obtain

$$
Q_{s}^{1} \circ Q_{1}^{s}+\mathrm{id}+h_{s} \circ \partial_{s}+\partial_{s} \circ h_{s}=0 .
$$


Then we can say $V_{s}^{1} \circ V_{1}^{s}=$ id. First the end points of type 1 are distinguished from Lemma 4.5. Second the end points of type 2 are constant maps because if they are not constant then they have non-zero dimension by the $R$-action. And constant maps are obviously distinguished. Finally we show that the end points of type 3 and 4 are distinguished. $\widetilde{F}_{s}\left(x_{+}\right)$.

Lemma 4.8. If $u \in \mathcal{M}_{\pi_{\omega}}\left(x_{-}, x_{+}\right)$, then we obtain $l(u) \geq \widetilde{F}_{s}\left(x_{-}\right)-$

Proof. We have $h(u) \leq a_{+}-a_{-}$in the same way as Lemma 4.7. And

$$
\begin{aligned}
l(u)=A(u)-l(u) & \geq a_{+}-a_{-} \\
& >\left(c_{+}-a_{+}\right)-\left(c_{-}-a_{-}\right)-C^{\prime} \\
& \geq \widetilde{F}_{s}\left(x_{-}\right)-\widetilde{F}_{s}\left(x_{+}\right)-C^{\prime} .
\end{aligned}
$$

Moreover $l(u)=\widetilde{F}_{s}\left(x_{-}\right)-\widetilde{F}_{s}\left(x_{+}\right)+n C^{\prime}, n \in Z$. Hence $l(u) \geq \widetilde{F}_{s}\left(x_{-}\right)-$ $\widetilde{F}_{s}\left(x_{+}\right)$.

In the case of type 3 , put $l\left(u_{1}\right)=\widetilde{F}_{s}(x)-\widetilde{F}_{s}(y)+n C^{\prime}$ and $l\left(u_{2}\right)=\widetilde{F}_{s}(y)-$ $\widetilde{F}_{s}(z)-n C^{\prime}$. Since $l\left(u_{1}\right)=A\left(u_{1}\right) \geq 0$, we obtain $n \geq 0$. From Lemma 4.8, $l\left(u_{2}\right) \geq \widetilde{F}_{s}(y)-\widetilde{F}_{s}(z)$, we obtain $n \leq 0$. Hence $n=0$ and $u_{1}, u_{2}$ are distinguished. Similarly, in the case of type 4 , put $l\left(u_{1}\right)=\widetilde{F}_{s}(x)-\widetilde{F}_{s}(y)+n C^{\prime}$ and $l\left(u_{2}\right)=\widetilde{F}_{s}(y)-\widetilde{F}_{s}(z)-n C^{\prime}$. Since $l\left(u_{2}\right)=A\left(u_{2}\right) \geq 0$, we obtain $n \leq 0$. From Lemma $4.8, l\left(u_{1}\right) \geq \widetilde{F}_{s}(x)-\widetilde{F}_{s}(y)$, we obtain $n \geq 0$. Hence $n=0$ and $u_{1}, u_{2}$ are distinguished.

Then we obtain $V_{s}^{1} \circ V_{1}^{s}=$ id.

\section{Proof of main theorem}

We summarize our story. Let $M$ be a closed contact manifold with a contact form $\gamma$. Let $L_{0}$ be a closed pre-Lagrangian submanifold of $M$ and $L_{1}$ a closed Legandrian submanifold of $M$ which satisfy the conditions $L_{0} \supset L_{1}$ and the boundary homomorphism $\pi_{2}\left(M, L_{0}\right) \rightarrow \pi_{1}\left(L_{0}\right)$ is trivial. We take $\widehat{L_{0}}$ the Lagrangian lift of $L_{0}$ with the condition $\min _{p \in \widehat{L_{0}}} \theta(p)=0$.

We denote the symplectization of $M$ by $P$. Let $\widetilde{H}_{s}$ be a time-dependent Hamiltonian function on $P$, and $G^{s}: P \rightarrow P$ the time-s flow generated by $\widetilde{H}_{s}$. We put

$$
a_{+}:=\int_{0}^{1} \max _{p \in G^{s}\left(\widehat{L_{0}}\right)} \widetilde{H}_{s}(p) d s \text { and } a_{-}:=\int_{0}^{1} \min _{p \in G^{s}\left(\widetilde{L}_{0}\right)} \widetilde{H}_{s}(p) d s .
$$

Suppose that $C^{\prime} e^{d+\varepsilon}<C_{\gamma}\left(L_{1}\right)$, where $d=\int_{0}^{1} \max _{p \in G^{t}\left(\widehat{L_{0}}\right)}\left|d \theta\left(X_{\widetilde{H}_{t}}\right)\right| d t$, then we can define Floer homology. Moreover if we have $a_{+}-a_{-}<C^{\prime}$, then there are homomorphisms $V_{1}^{s}: H\left(C(s), \partial_{s}\right) \rightarrow H\left(C(1), \partial_{1}\right)$ and $V_{s}^{1}: H\left(C(1), \partial_{1}\right) \rightarrow$ 
$H\left(C(s), \partial_{s}\right)$, for small $s$, such that $V_{s}^{1} \circ V_{1}^{s}=$ id. Hence if $G^{1}\left(\widehat{L_{0}}\right)$ intersects $R \times L_{1}$ transversaly, we obtain

$$
\begin{aligned}
\sharp\left\{G^{1}\left(\widehat{L_{0}}\right) \cap\left(R \times L_{1}\right)\right\} & \geq \operatorname{rank} H\left(C(1), \partial_{1}\right) \\
& \geq \operatorname{rank} H\left(C(s), \partial_{s}\right) .
\end{aligned}
$$

And, for small $s, H\left(C(s), \partial_{s}\right)$ is isomorphic to $H_{*}\left(L_{1} ; Z_{2}\right)$ as a vector space. Hence we obtain

$$
\sharp\left\{G^{1}\left(\widehat{L_{0}}\right) \cap\left(R \times L_{1}\right)\right\} \geq \operatorname{rank} H\left(L_{1} ; Z_{2}\right) .
$$

In the next step, let $H_{s}$ be a time-dependent function on $M$ and $X_{H_{s}}$ the contact vector field generated by $H_{s}$. Let $e^{\theta} H_{s}$ be a Hamiltonian function on $P$, then the Hamiltonian vector field generated by $e^{\theta} H_{s}$ is $\left(d H_{s}(Y), X_{H_{s}}\right)$ on $P=$ $R \times M$, where $Y$ is the Reeb vector field. We put $d=\max _{s \in[0,1], p \in M}\left|d H_{s}(Y)\right|$ and moreover

$$
a_{+}:=\int_{0}^{1} \max _{p \in M} H_{s}(p) d s \cdot \exp \left\{d+h\left(\widehat{L_{0}}\right)\right\}
$$

and

$$
a_{-}:=\int_{0}^{1} \min _{p \in M} H_{s}(p) d s \cdot \exp \left\{d+h\left(\widehat{L_{0}}\right)\right\} .
$$

Finally, if we have $C^{\prime} e^{d+\varepsilon}<C_{\gamma}\left(L_{1}\right)$ and $a_{+}-a_{-}<C^{\prime}$, then Theorem 1.1 holds.

\section{Compactness}

For simplicity we fix the almost complex structure $\tilde{J}$ on $P$ defined in the introduction. To put it more precisely, we have to consider perturbations of almost complex structures. It is a little complicated. Hence we omit it here. See [2].

We introduce notation. Put $K_{a, b}:=[a, b] \times M \subset P$ and $M_{\theta}:=\{\theta\} \times M$. If we denote $d=\int_{0}^{s} \max _{p \in G^{t}\left(\widehat{L_{0}}\right)}\left|d \theta\left(X_{\widetilde{H}_{t}}\right)\right| d t$, then we may have $G^{s}\left(\widehat{L_{0}}\right) \subset$ $K_{-d, h\left(L_{0}\right)+d+\varepsilon}$.

Lemma 6.1. For any $u \in \mathcal{M}_{s}\left(x_{-}, x_{+}\right)$, the image of $u$ is contained in $K_{-\infty, h\left(L_{0}\right)+d+\varepsilon \text {. }}$

Proof. Assume that the image of $u$ is not contained in $K_{-\infty, h\left(L_{0}\right)+d+\varepsilon}$, then we have $\sup (\theta \circ u)>h\left(L_{0}\right)+d+\varepsilon$. Because $u$ converges to $x_{ \pm}$at infinity and $\theta\left(x_{ \pm}\right) \leq h\left(L_{0}\right)+d+\varepsilon$, there are some points of the image of $u$ where $\theta \circ u$ takes the maximum. Let $p_{0}$ be one of these points. From the pseudoconvexity of $M_{\theta}$ and maximum principle, $p_{0}$ is not an interior point of the image of $u$. Assume that $p_{0}$ is a boundary point. Let $v$ be a tangent vector 
along the boundary at $p_{0}$. From $p_{0} \in K_{h\left(L_{0}\right)+d+\varepsilon, \infty}$, we have $p_{0} \in R \times L_{1}$ and $v$ is tangent to $L_{1}$. Then $v \in \xi_{p_{0}}$. Because $\tilde{J} v \in \tilde{J} \xi_{p_{0}}=\xi_{p_{0}}$ and $u$ is pseudoholomorphic, the image of $u$ is tangent to $M_{\theta\left(p_{0}\right)}$ at $p_{0}$. But this contradicts the pseudo-convexity of $M_{\theta}$ and strong maximum principle.

We denote the pull-back of $\gamma$ by the projection $\pi: R \times M \rightarrow M$, also by $\gamma$. Let $\bar{\omega}:=d \gamma$, then we have $\left.\bar{\omega}\right|_{T_{p}(R \times M)}=e^{-\theta}(d \pi)^{*}\left(\left.\omega\right|_{T_{p} M_{\theta}}\right)$, where $p \in M_{\theta}$.

Lemma 6.2. Put $C^{i}:=u^{-1}\left(K_{-\infty,-i}\right)$ for $u \in \mathcal{M}_{s}\left(x_{-}, x_{+}\right)$. If $i \geq d$, then we have

$$
0 \leq \int_{C^{i}} u^{*} \bar{\omega}=e^{i} \int_{C^{i}} u^{*} \omega .
$$

Hence, if $\int_{R \times[0,1]} u^{*} \omega<C^{\prime}$, then we obtain $0 \leq \int_{C^{i}} u^{*} \bar{\omega}<C^{\prime} e^{i}$.

Proof. From $i \geq d, \partial C^{i}=u^{-1}\left(M_{-i}\right) \cup u^{-1}\left(\left(R \times L_{1}\right) \cap K_{-\infty,-i}\right)$. Notice that $L_{1}$ is a Legandrian submanifold. Hence

$$
\int_{C^{i}} u^{*} \bar{\omega}=\int_{\partial C^{i}} u^{*} \gamma=\int_{u^{-1}\left(M_{-i}\right)} u^{*} \gamma=e^{i} \int_{u^{-1}\left(M_{-i}\right)} u^{*}\left(e^{\theta} \gamma\right)=e^{i} \int_{C^{i}} u^{*} \omega .
$$

Corollary 6.3. If $i \geq d$, then we have

$$
\int_{C^{i}} u^{*} \omega \leq e^{d-i} \int_{C^{d}} u^{*} \omega
$$

Hence, if $\int_{R \times[0,1]} u^{*} \omega<C^{\prime}$, then we obtain $\int_{C^{i}} u^{*} \omega<C^{\prime} e^{d-i}$.

Proof. Since $u$ is pseudo-holomorphic, $\int_{C^{i+1}} u^{*} \bar{\omega} \leq \int_{C^{i}} u^{*} \bar{\omega}$. Then from Lemma 6.2

$$
e^{i+1} \int_{C^{i+1}} u^{*} \omega=\int_{C^{i+1}} u^{*} \bar{\omega} \leq \int_{C^{i}} u^{*} \bar{\omega}=e^{i} \int_{C^{i}} u^{*} \omega .
$$

Hence we obtain

$$
\int_{C^{i+1}} u^{*} \omega \leq e^{-1} \int_{C^{i}} u^{*} \omega
$$

and repeat this inequality.

For a map $u: R \times[0,1] \rightarrow P$, if a domain $G$ of $R \times M$ satisfies the following conditions, we call $G$ a special domain for $u$ of level $k$ and width $l$.

- $G$ is either a disk or an annulus.

- $\left.u\right|_{G}$ intersects $M_{-k} \cup M_{-k-l}$ transversaly.

- $u(\partial G) \subset M_{-k} \cup M_{-k-l} \cup\left(R \times L_{1}\right)$ and $u(\partial G \cap \partial(R \times[0,1])) \subset R \times L_{1}$.

- $u(\partial G) \cap M_{-k} \neq \emptyset$ and $u(\partial G) \cap M_{-k-l} \neq \emptyset$.

- $u(G) \subset K_{-\infty,-d}$. 
Lemma 6.4. For $u \in \mathcal{M}_{s}\left(x_{-}, x_{+}\right), C^{i}$ is a disjoint union of disks.

Proof. It follows from the pseudo-convexity of $M_{\theta}$ and maximum principle.

Again note that, for simplicity we fix the almost complex structure $\tilde{J}$ on $P$ defined in the introduction. To put it more precisely, we have to consider perturbations of almost complex structures. It is a little complicated. Hence we omit it here. See [2].

Lemma 6.5. Let $\left\{u_{n}\right\} \subset \mathcal{M}_{s}\left(x_{-}, x_{+}\right)$be a sequence of pseudo-holomorphic maps. Assume that the union of the images of $u_{n}{ }^{\prime} s$ is not contained in any compact subset of $P$. Then there are a subsequence $\left\{u_{n_{k}}\right\}$ and a sequence of domains $\left\{G_{k}\right\}, G_{k} \subset R \times[0,1]$, which satisfy the following.

- $G_{k}$ is a special domain for $u_{n_{k}}$.

- $G_{k}$ is of width $l$ and level $j$ for $d \leq j$ and $u_{n_{k}}\left(G_{k}\right) \subset K_{-j-2 l,-j}$.

- If $\int_{R \times[0,1]} u_{n}^{*} \omega<C^{\prime}$, then we have $\int_{G_{k}} u_{n_{k}}^{*} \bar{\omega} \rightarrow 0$ as $k \rightarrow \infty$.

Proof. From Lemma 6.1, the union of the images of $u_{n}{ }^{\prime} s$ is bounded above along the $R$-direction of $P$. Hence we may assume that there is a subsequence $\left\{u_{n_{k}}\right\}$ such that $u_{n_{k}}(R \times[0,1]) \cap M_{-(k+1) l,-d} \neq \emptyset$. For $d \leq i \leq d+k l$ we put

$$
B_{k}^{i}:=u_{n_{k}}^{-1}\left(K_{-\infty,-i}\right) \backslash u_{n_{k}}^{-1}\left(K_{-\infty,-i-l}\right) .
$$

Let $B$ be a connected component of $B_{k}^{i}$ such that $u_{n_{k}}^{-1}\left(M_{-i}\right) \cap B \neq \emptyset$ and $u_{n_{k}}^{-1}\left(M_{-i-l}\right) \cap B \neq \emptyset$. From Lemma $6.4, B$ is a disk with some holes. We patch some disks back to these holes so that $B$ turns to either a disk or an annulus. We denote this disk or annulus by $\widehat{B}$. Especially we can do this procedure so that $\partial \widehat{B} \cap u_{n_{k}}^{-1}\left(M_{-i}\right) \neq \emptyset$ and $\partial \widehat{B} \cap u_{n_{k}}^{-1}\left(M_{-i-l}\right) \neq \emptyset$. Then $\widehat{B}$ is a special domain for $u_{n_{k}}$ of width $l$ and level $i$. In this way we can find special domains.

For each $k$, we can find special domains $\widehat{B}_{k}^{j}$ for $u_{n_{k}}$ of width $l$ and level $j$, $j=d, d+l, \ldots, d+k l$, such that Int $\widehat{B}_{k}^{i} \cap$ Int $\widehat{B}_{k}^{j}=\emptyset$ for $i \neq j$. From $\bigcup_{j} \widehat{B}_{k}^{j} \subset u_{n_{k}}^{-1}\left(K_{-\infty,-d}\right)$,

$$
\sum_{j} \int_{\widehat{B}_{k}^{j}} u_{n_{k}}^{*} \bar{\omega} \leq \int_{C_{k}^{d}} u_{n_{k}}^{*} \bar{\omega}=e^{d} \int_{C_{k}^{d}} u_{n_{k}}^{*} \omega,
$$

where $C_{k}^{d}=u_{n_{k}}^{-1}\left(K_{-\infty,-d}\right)$. Hence, if $\int_{R \times[0,1]} u_{n}^{*} \omega<C^{\prime}$, we have

$$
\sum_{j} \int_{\widehat{B}_{k}^{j}} u_{n_{k}}^{*} \bar{\omega}<C^{\prime} e^{d}
$$

Because each term of the sum is positive, there is at least one special domain $\widehat{B}_{k}^{j}$ such that $\int_{\widehat{B}_{k}^{j}} u_{n_{k}}^{*} \bar{\omega}<C^{\prime} e^{d} / k$. 
We put a special domain $G_{k}$ for $u_{n_{k}}$ such that $\int_{G_{k}} u_{n_{k}}^{*} \bar{\omega}$ is the minimum over all the special domains of width $l$ and level $d, d+l, \ldots, d+k l$. From the construction, we have $G_{k} \cap M_{-j+l}=\emptyset$, where $j$ is the level of $G_{k}$. Moreover we have $G_{k} \cap M_{-j-2 l}=\emptyset$, because if $G_{k} \cap M_{-j-2 l} \neq \emptyset$ we can find another special domain $G_{k}^{\prime}$ such that $\int_{G_{k}^{\prime}} u_{n_{k}}^{*} \bar{\omega}<\int_{G_{k}} u_{n_{k}}^{*} \bar{\omega}$. This contradicts the minimum of $G_{k}$.

Fix a width $l$. We put $\widetilde{u}_{n_{k}}$ to be the $(j-d)$-shift, along the $R$-direction of $P$, of a pseudo-holomorphic map $u_{n_{k}}: G_{k} \rightarrow P$, where $j$ is the level of $G_{k}$. Then we have

$$
\int_{G_{k}} \widetilde{u}_{n_{k}}^{*} \omega=e^{j-d} \int_{G_{k}} u_{n_{k}}^{*} \omega
$$

From the inequality $\int_{u_{n_{k}}^{-1}\left(K_{-\infty,-j}\right)} u_{n_{k}}^{*} \omega \leq e^{d-j} \int_{u_{n_{k}}^{-1}\left(K_{-\infty,-d}\right)} u_{n_{k}}^{*} \omega$, in Corollary 6.3 , we obtain

$$
\int_{G_{k}} \widetilde{u}_{n_{k}}^{*} \omega \leq e^{j-d} \int_{u_{n_{k}}^{-1}\left(K_{-\infty,-j}\right)} u_{n_{k}}^{*} \omega \leq \int_{u_{n_{k}}^{-1}\left(K_{-\infty,-d}\right)} u_{n_{k}}^{*} \omega .
$$

Hence, if $\int_{R \times[0,1]} u_{n_{k}}^{*} \omega<C^{\prime}$, then we have

$$
\int_{G_{k}} \widetilde{u}_{n_{k}}^{*} \omega<C^{\prime}
$$

Consider the pseudo-holomorphic maps $\widetilde{u}_{n_{k}}: G_{k} \rightarrow(-d-2 l,-d] \times M$, and apply the Gromov's compactness theorem. See [2].

Proposition 6.6. There is a subsequence $\left\{\widetilde{u}_{n_{k}}\right\}$ which converges uniformly on compact sets to a non-constant pseudo-holomorphic map $\widetilde{u}_{\infty}$. The boundary of this image is contained in $M_{-d} \cup M_{-d-l} \cup\left(R \times L_{1}\right)$ and smoothness of the boundary holds at points in $R \times L_{1}$.

Notice that, if $\int_{R \times[0,1]} u_{n_{k}}^{*} \omega<C^{\prime}$, we have $\int_{G_{k}} \widetilde{u}_{n_{k}}^{*} \bar{\omega} \rightarrow 0$ as $k \rightarrow \infty$. Hence we obtain $\int_{B} \widetilde{u}_{\infty}^{*} \bar{\omega}=0$, where $B$ is either a disk or an annulus.

Lemma 6.7. Let $\widetilde{u}_{\infty}: \operatorname{Int} B \rightarrow(-d-2 l,-d] \times M$ be a non-constant pseudo-holomorphic map, where $B$ is either a disk or an annulus, and the boundary of this image be contained in $M_{-d} \cup M_{-d-l} \cup\left(R \times L_{1}\right)$. Assume that $\int_{B} \widetilde{u}_{\infty}^{*} \bar{\omega}=0$. Then there is either a closed orbit of the Reeb vector field or an orbit of the Reeb vector field with the end points in $L_{1}$, we denote each by $S$, such that

$$
\widetilde{u}_{\infty}(\operatorname{Int} B)=(-d-l,-d) \times S \subset(-d-l,-d) \times M .
$$

And we obtain

$$
e^{-d}\left(1-e^{-l}\right) \int_{S} \gamma=\int_{B} \widetilde{u}_{\infty}^{*} \omega
$$


Proof. From $\bar{\omega}=d \gamma, \omega=e^{\theta}(d \theta \wedge \gamma+d \gamma)$ and $\widetilde{u}_{\infty}$ is pseudo-holomorphic, this lemma holds.

If $\int_{R \times[0,1]} u_{n}^{*} \omega<C^{\prime}$, then we have $\int_{G_{k}} \widetilde{u}_{n}^{*} \omega<C^{\prime}$ as mentioned after Lemma 6.5. Hence $\int_{B} \widetilde{u}_{\infty}^{*} \omega<C^{\prime}$ and we obtain

$$
\int_{S} \gamma<e^{d}\left(1-e^{-l}\right)^{-1} C^{\prime}
$$

If we have $C^{\prime} e^{d+\varepsilon}<C_{\gamma}\left(L_{1}\right)$ for $\varepsilon>0$, all the images of $u_{n}$ have to be contained in a compact set of $P$ from Lemma 6.5, Proposition 6.6 and Lemma 6.7.

Thus we finish a proof of Theorem 3.1.

Acknowledgement. The author wishes to thank K. Fukaya for his encouragement.

\author{
DEPARTMENT OF MATHEMATiCs \\ FACULTy OF SCIENCE \\ TOKyo Metropolitan University \\ HACHIOHJI 192-0397 JAPAN
}

\title{
References
}

[1] Y. V. Chekanov, Hofer's Symplectic Energy and Lagrangian intersections, in Contact and Symplectic Geometry, Cambridge, 1996, 296-306.

[2] Y. Eliashberg, H. Hofer and D. Salamon, Lagrangian intersectons in contact geometry, Geom. Funct. Anal., 5-2 (1995), 244-269.

[3] A. Floer, Morse theory for Lagrangian intersections, J. Diff. Geom., 28 (1988), 513-547.

[4] H. Hofer, Pseudoholomorphic curves in symplectizations with applications to the Weinstein conjecture in dimention three, Invent. Math., 114 (1993), $515-563$. 